\title{
A Field-Effect Transistor Based on Cumulenic sp-Carbon Atomic Wires
}

\author{
Alberto D. Scaccabarozzi, Alberto Milani, Sonia Peggiani, Stefano Pecorario, Bozheng Sun, \\ Rik R. Tykwinski, Mario Caironi,* and Carlo S. Casari*
}

Cite This: J. Phys. Chem. Lett. 2020, 11, 1970-1974

Read Online

ABSTRACT: Carbyne and linear carbon structures based on sp-hybridization are attractive targets as the ultimate one-dimensional system (i.e., one-atom in diameter) featuring wide tunability of optical and electronic properties. Two possible structures exist for sp-carbon atomic wires: (a) the polyynes with alternated single-triple bonds and (b) the cumulenes with contiguous double bonds. Theoretical studies predict semiconducting behavior for polyynes, while cumulenes are expected to be metallic. Very limited experimental work, however, has been directed toward investigating the electronic properties of these structures, mostly at the single-molecule or monolayer level. However, sp-carbon atomic wires hold great potential for solution-processed thinfilm electronics, an avenue not exploited to date. Herein, we report the first field-effect transistor (FET) fabricated employing cumulenic sp-carbon atomic wires as a semiconductor material. Our proof-of-concept FET device is easily fabricated by solution drop casting and paves the way for exploiting sp-carbon atomic wires as active electronic materials.

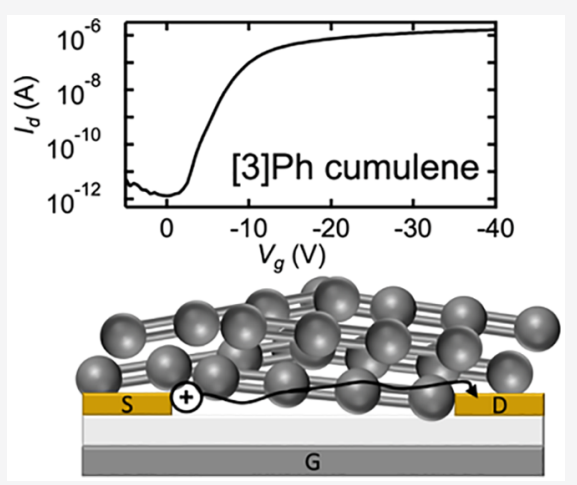

arbon nanostructures, including graphene and nanotubes, $\checkmark$ are widely investigated ${ }^{1-7}$ for electronic and optoelectronic applications in a "more-than-Moore" approach, as shown in the very recent fabrication of a fully functional microprocessor based on 14000 carbon-nanotube FETs. ${ }^{8}$ In addition to large-scale integration, these materials show appealing prospects for low-cost, flexible, stretchable, and wearable electronics. ${ }^{9}$ Difficulties in controlling chirality in nanotubes and introducing a band gap in graphene, however, have made implementation problematic. Beyond these more common carbon materials, the linear sp-carbon allotrope carbyne (or carbon atomic wire) has recently been considered as a new player in the family of carbon compounds as a result of outstanding properties that have been predicted by theoretical studies. ${ }^{2,7,10,11}$ In particular, ballistic and oscillatory conductance, ${ }^{12}$ spin-dependent transport, ${ }^{13}$ and a straininduced metal-to-insulator transition ${ }^{4}$ are predicted in spcarbon atomic wires, making these systems promising candidates for the future of "organic" electronics. The application of carbon atomic wires in electronics also shares the processing advantages of $\mathrm{sp}^{2}$-conjugated organic molecules, toward scalable, solution-based large-area manufacturing, while offering higher intrinsic electronic performances. Despite these expectations, the exploitation of carbon atomic wires has been challenged by issues centered on synthesis and chemical stability. ${ }^{14,15}$ As a result, sp-carbon structures are often viewed as exotic, elusive systems in spite of their potential. In fact, the experimental synthesis of stable carbon atomic wires has always faced difficulties, particularly in the case of cumulenes, which are considerably less stable than polyynes because of the onset of Peierls' distortion.

There is an increasing number of theoretical reports focusing on the electronic and transport properties of carbon atomic wires, ${ }^{13,16}$ but experimental studies are scarce and mostly involve single molecules or molecular mononlayer ensembles. ${ }^{17-20}$ Transport measurements have been performed on single carbon atomic wires, recording a lower conductance than predicted, attributed to contact resistance effects. ${ }^{20} \mathrm{~A}$ carbon wire suspended between graphene edges has been fabricated in a transmission electron microscopy experiment and electrically characterized in situ, displaying a short lifetime even in vacuum. ${ }^{4,5}$ A two-terminal memristor based on the voltage-induced formation and rupture of carbon atomic wires in a graphene-based device has also been proposed. ${ }^{6,7}$ Finally, studies have very recently shown intriguing behavior for cumulene molecular wires. ${ }^{17}$ On the other hand, materials based on carbon wires are rare, as is the characterization of their thin-film electrical properties. ${ }^{21}$ Attempts to integrate films of carbon wires in electrical devices have been, to our knowledge, not reported to date.

Received: January 14, 2020

Accepted: February 18, 2020

Published: February 18, 2020 
As a first step toward exploiting sp-carbon-based materials in thin-film devices, we demonstrate herein the realization of a field-effect transistor (FET) based on a cumulenic carbon atomic wire. While a cumulenic structure can refer to an infinite structure of contiguous double bonds (a metal), the definition used herein is that of a molecule containing three or more contiguous double bonds (a semiconductor). ${ }^{22,23}$ A FET has been selected because it serves as a test-bed for the electronic properties of cumulenes as an active material (through investigation of charge injection and transport), and because it holds a direct technological relevance. Toward this goal, the targeted sp-carbon atomic wire must fulfill several challenging requirements, including semiconducting behavior, suitably low energy gap to facilitate charge injection, solution processability, and stability under ambient conditions. While polyynes are semiconducting, the longest derivatives show a quite large gap (exceeding $3 \mathrm{eV}$ for $20 \mathrm{sp}$-carbon atoms), ${ }^{24}$ and processing is challenging. Hence, the choice has been directed to a cumulenic system, a [3]cumulene, in which end groups contribute to bond length alternation, ensuring a semiconducting material featuring an energy gap in the visible region of the spectrum.

Tetraphenylbutatriene $([3] \mathrm{Ph})$ has been chosen as the molecular semiconductor, consisting of a short carbon wire composed of three cumulated double bonds (i.e., four carbon atoms) and terminated at each end by two phenyl moieties (Figure $1 \mathrm{~b}$ ). [3] $\mathrm{Ph}$ is colored with an absorption onset in

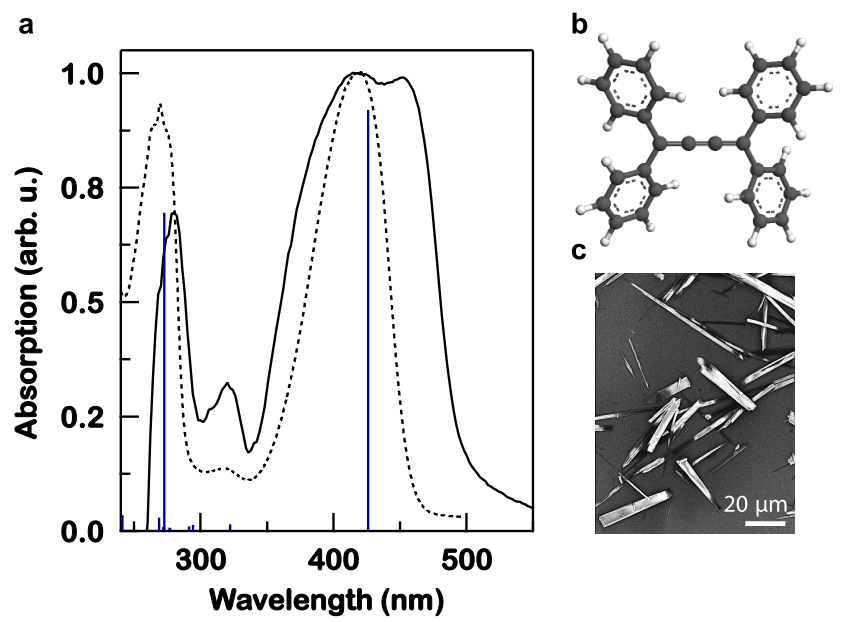

Figure 1. (a) UV-vis absorption spectra of [3] $\mathrm{Ph}$ in dichloromethane solution (dashed black line) and drop-cast solid-state deposit (solid black line). The TD-DFT-computed single-molecule transitions are shown in blue lines. (b) Molecular structure of [3] Ph and (c) SEM image of the elongated crystals forming upon solution drop casting.

dichloromethane (DCM) solution at about $460 \mathrm{~nm}$ corresponding to a DFT-computed highest occupied molecular orbital-lowest occupied molecular orbital (HOMO-LUMO) energy gap of $3.2 \mathrm{eV}$. The cumulenic nature of $[3] \mathrm{Ph}$ is reflected in an experimental bond-length alternation (BLA) of $0.088 \AA{ }^{25}$ very close to the theoretical prediction of $0.086 \AA$, significantly different from the typical BLA of polyyne structures (e.g., exceeding $0.14 \AA$ for diphenyl polyynes with four carbon atoms). ${ }^{26}$ Using simple solution drop casting (4 $\mathrm{mg} / \mathrm{mL}$ in DCM), [3] $\mathrm{Ph}$ molecules assemble in the solid state to form long needle-like crystals (up to several hundreds of micrometers) as shown in the scanning electron microscopy
(SEM) image (Figure 1c). The crystals are stable under ambient conditions and up to $\sim 250{ }^{\circ} \mathrm{C}$ (differential scanning calorimetry (DSC) measurements are reported in the Supporting Information, Figure S1). Crystals of [3] $\mathrm{Ph}$ show a red-shifted UV-vis absorption when compared to their solution counterpart and exhibit a new absorption centered at around $450 \mathrm{~nm}$ (Figure 1a). This structured absorption is of vibronic origin, as previously suggested, ${ }^{27}$ and reflects a solidstate intermolecular interaction within the macroscopic crystals. As shown in Figure 1a, TD-DFT calculations predict excited states with significant oscillator strength in very good correlation with the experimental absorption spectra (more details are provided in the Supporting Information).

The needle-like structures formed upon solution casting exhibit sharp diffraction patterns (Figure 2b) that are in
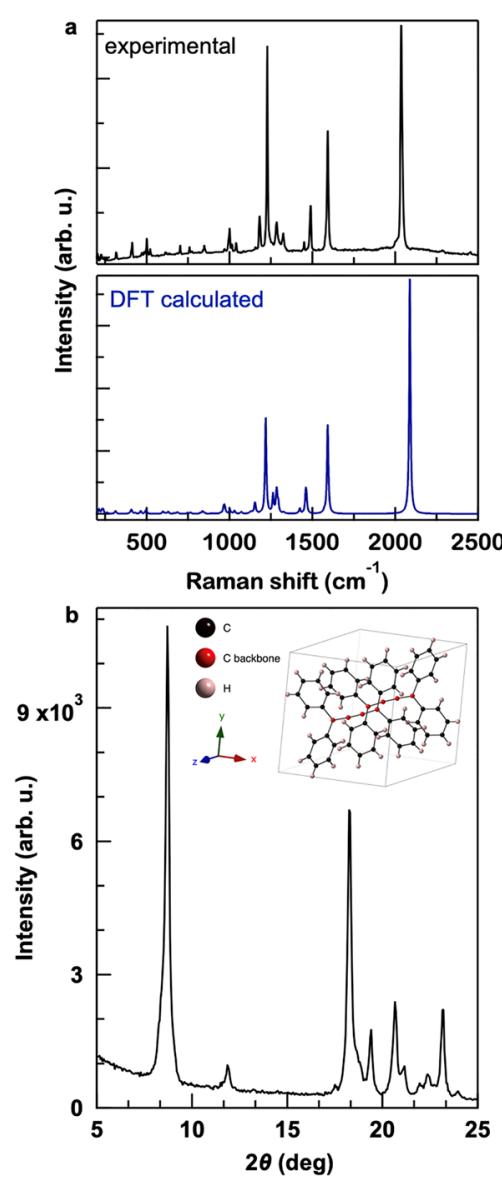

Figure 2. (a) Experimental Raman spectrum of [3] Ph taken at 632 $\mathrm{nm}$ on the solid sample (top panel) and DFT calculations (PBE0-D2/ $6-31 G(d, p)$ ) of the Raman spectrum of the crystal (bottom panel). (b) XRD diffractogram of the drop-cast deposit. The inset shows the unit cell of crystalline [3] Ph.

agreement with the crystallographic data reported for single crystals of tetraphenylbutatriene. ${ }^{28,29}$ The unit cell is reported to be triclinic; the steric interactions between phenyl groups preclude complete planarity, and hence, face-to-face phenyl packing is prevented. Nevertheless, a very dense molecular packing occurs, with arrays of molecules in contact along the carbon chain. The cumulenic nature of $\mathrm{C}=\mathrm{C}$ bonds is retained in the solid state; indeed, Raman spectroscopy measurements (Figure 2a) unambiguously identify sp-carbon atomic wires by revealing the characteristic fingerprint of sp-carbon at about 
$2000 \mathrm{~cm}^{-1}$ for [3] Ph (effective conjugation coordinate (ECC) mode consisting in an oscillation of the BLA). ${ }^{30}$ Features related to the terminal phenyl groups are found at $1600 \mathrm{~cm}^{-1}$, corresponding to the stretching of the $\mathrm{CC}$ bond in the aryl ring. DFT calculations of the Raman response are in nice agreement with experiments, as demonstrated in previous studies. $^{2}$ A detailed assignment of the Raman spectra based on DFT calculations of the crystal are reported in the Supporting Information.

We investigated the charge accumulation properties of the cast cumulenic [3] Ph microcrystals in FETs. Devices were fabricated employing a bottom-gate bottom-contact configuration, based on interdigitated Au source and drain contacts, with a channel width $(W)$ of $1 \mathrm{~cm}$, channel lengths $(L)$ ranging from 2.5 to $20 \mu \mathrm{m}, \mathrm{SiO}_{2}$ as the dielectric, and highly doped silicon substrate as gate electrode (Figure $3 \mathrm{c}$ ). Gold electrodes

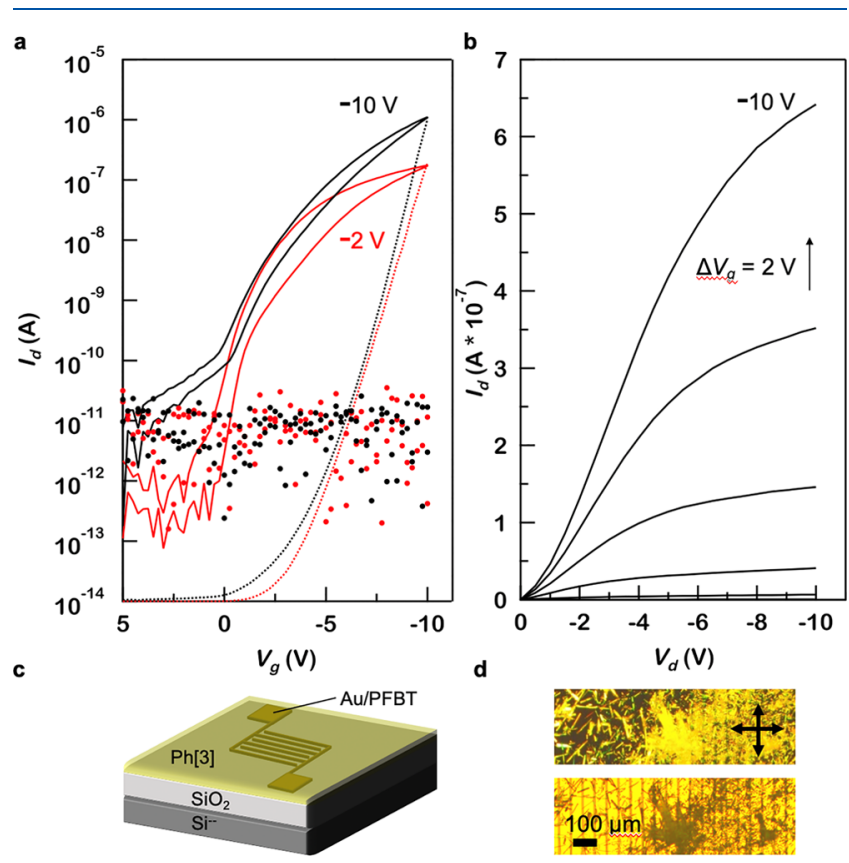

Figure 3. (a) Representative transfer characteristic of a [3]Ph microcrystal bottom-gate bottom-contact field effect transistor $(L=5$ $\mu \mathrm{m} ; W=1 \mathrm{~cm}$ ). Drain voltages in linear (red curve) and saturation (black curve) regimes are indicated with a solid line. The $I_{\mathrm{d}}$ is also shown in a linear and a square root scale for linear and saturation regime, respectively (dotted line), while the gate current is shown as a dotted line. (b) Corresponding output characteristic. The gate voltage is indicated. (c) Device architecture and (d) optical micrographs of the FET in bright field (bottom) and polarized light (top).

were functionalized with a pentafluorobenzenethiol (PFBT) self-assembled monolayer (SAM), commonly employed in organic-based electronic devices to increase the electrode work function to favor hole injection. ${ }^{31}$ A solution of $[3] \mathrm{Ph}(4 \mathrm{mg} /$ $\mathrm{mL}$ in DCM) was drop cast onto the electrodes and dielectric as the last step. These devices enabled the very first observation of a p-type field-effect behavior for a cumulenic derivative, i.e., a modulation of the conductivity of microcrystals bridging the electrodes upon the accumulation of holes (Figure $3 \mathrm{a}-\mathrm{b}$ ). Clear source-to-drain current $\left(I_{\mathrm{d}}\right)$ modulation is achieved by sweeping the gate voltage $\left(V_{\mathrm{g}}\right)$, with an $I_{\text {on }} / I_{\text {off }}$ ratio of $10^{5}-10^{6}$. Good square root (linear) behavior of the current as a function of gate voltage in the saturation (linear) regime is observed. Gate leakage is negligible, while a relatively steep subthreshold slope $(750 \mathrm{mV} / \mathrm{dec})$ is measured. We have fit the $I_{\mathrm{d}}$ characteristic curve in the range where it shows either a linear dependence with $V_{\mathrm{g}}$, for $V_{\mathrm{d}}=-2 \mathrm{~V}$, or a quadratic dependence, for $V_{\mathrm{d}}=-10 \mathrm{~V}$, to extract the linear and saturation field-effect mobility $(\mu)$, respectively. ${ }^{32}$ Charge carrier mobility is a crucial parameter in semiconducting materials, because it describes how fast charges move in the semiconductor upon application of an external field; hence, it is directly related to the performance of electronic devices. A determination of $\mu$ in the reported FETs is complicated by the incomplete and nonuniform coverage of the device active area, affecting the real channel width actually contributing to charge accumulation and transport, with respect to the geometrical one, $W$. In fact, [3] Ph tends to form elongated crystallites that are randomly distributed on the substrate, crossing the channel nonuniformly at various angles (Figure $3 \mathrm{~d}$ ). If the full geometrical width of the electrodes is adopted for the extraction of the mobility, a conservative value of $2 \times 10^{-3}$ $\mathrm{cm}^{2} /(\mathrm{V} \mathrm{s})$ is determined. Considering the poor film coverage of the semiconductor in the device, this value is certainty a lower limit of the true mobility. Moreover, output characteristics, where a slight S-shape at low voltages is visible, show how contact resistance represents a limiting factor, despite the PFBT contact treatment. As such, we confidently predict that future engineering of the deposition techniques will increase coverage and improve charge injection, offering optimized films and higher charge mobilities.

In conclusion, we have established that cumulenic sp-carbon materials can be easily processed from solution to fabricate well-behaving p-type field-effect transistors. The investigated molecules are short carbon wires that feature a cumulene structure, as confirmed by the low BLA of ca. $0.08 \AA$. Solution casting of these sp-molecules gives needle-like microcrystals that are stable at ambient conditions and allows the first assessment of the electronic performance as active material in a transistor device. In spite of its simplicity, this approach to organic devices based on sp-carbon molecules has not been previously explored, and yet it holds a great scientific and technological potential. This work should provide a new realm for semiconductor materials science research, toward the employment of sp-atomic wires in the realization of a new generation of solution-processable, all-carbon optoelectronic devices.

\section{ASSOCIATED CONTENT}

\section{Supporting Information}

The Supporting Information is available free of charge at https://pubs.acs.org/doi/10.1021/acs.jpclett.0c00141.

Methods; DSC results of [3] Ph (Figure S1); XRD diffractogram of the drop cast deposit and single crystal diffractions (Figure S2); $\mid I_{\mathrm{d}} \mathrm{d}^{1 / 2}$ as a function of $V_{\mathrm{g}}$ in saturation regime and $I_{\mathrm{d}}$ as a function of $V_{\mathrm{g}}$ in linear regime (Figure S3); field-effect mobility as a function of $V_{\mathrm{g}}$ (Figure S4); optical micrographs of FET device (Figures S5 and S6); FET parameters (Table S1); TDDFT-computed singlet excited states of [3] $\mathrm{Ph}$ single molecules (Table S2); DFT-computed values of vibrational frequency, IR intensity, and Raman activity and sketches of the normal modes associated with the most significant Raman bands of the [3] Ph crystal (Table S3) (PDF) 


\section{AUTHOR INFORMATION}

\section{Corresponding Authors}

Mario Caironi - Center for Nano Science and Technology@ PoliMi, Istituto Italiano di Tecnologia, Milan 20133, Italy; ○ orcid.org/0000-0002-0442-4439; Email: mario.caironi@ iit.it

Carlo S. Casari - Micro and Nanostructured Materials Lab NanoLab, Department of Energy, Politecnico di Milano, 20133 Milano, Italy; @ orcid.org/0000-0001-9144-6822; Email: carlo.casari@polimi.it

\section{Authors}

Alberto D. Scaccabarozzi - Center for Nano Science and Technology@PoliMi, Istituto Italiano di Tecnologia, Milan 20133, Italy

Alberto Milani - Micro and Nanostructured Materials Lab NanoLab, Department of Energy, Politecnico di Milano, 20133 Milano, Italy; @ orcid.org/0000-0001-6026-5455

Sonia Peggiani - Micro and Nanostructured Materials Lab NanoLab, Department of Energy, Politecnico di Milano, 20133 Milano, Italy

Stefano Pecorario - Center for Nano Science and Technology@ PoliMi, Istituto Italiano di Tecnologia, Milan 20133, Italy; Micro and Nanostructured Materials Lab NanoLab, Department of Energy, Politecnico di Milano, 20133 Milano, Italy

Bozheng Sun - Department of Chemistry, University of Alberta, Edmonton, Alberta, Canada T6G 2G2

Rik R. Tykwinski - Department of Chemistry, University of Alberta, Edmonton, Alberta, Canada T6G 2G2; 10 orcid.org/ 0000-0002-7645-4784

Complete contact information is available at:

https://pubs.acs.org/10.1021/acs.jpclett.0c00141

\section{Author Contributions}

C.S.C. and M.C. conceived the work. C.S.C. and A.D.S. wrote the manuscript draft. All authors reviewed and discussed the manuscript. A.D.S fabricated the field-effect transistors and performed the electrical measurements and parameter extraction. A.D.S performed UV-vis spectroscopy measurements and optical microscopy imaging. S. Pecorario performed the XRD measurements; A.D.S analyzed the data; A.M. carried out DFT calculations; S. Peggiani performed Raman measurements; S. Peggiani and S. Pecorario performed the SEM investigation. R.R.T and B.S. completed synthesis, purification, and thermal analysis of [3] Ph.

\section{Notes}

The authors declare no competing financial interest.

\section{ACKNOWLEDGMENTS}

A.S. and M.C. acknowledge funding from the European Research Council (ERC) under the European Union's Horizon 2020 research and innovation programme "HEROIC", Grant Agreement 638059. C.S.C, A.M., and S. Peggiani acknowledge funding from the European Research Council (ERC) under the European Union's Horizon 2020 research and innovation program ERC Consolidator Grant "EspLORE" (www.esplore.polimi.it), Grant Agreement No. 724610. R.R.T. is grateful for funding from the Natural Sciences and Engineering. This work has been partially carried out at Polifab, the micro- and nanotechnology center of the Politecnico di Milano. The authors acknowledge Mohamed
Tarek Said Zoelfakar Soliman and Alessandro Vidale for the preliminary work done during their Master Thesis.

\section{REFERENCES}

(1) Hirsch, A. The Era of Carbon Allotropes. Nat. Mater. 2010, 9, 868

(2) Casari, C. S.; Tommasini, M.; Tykwinski, R. R.; Milani, A. Carbon-Atom Wires: 1-D Systems with Tunable Properties. Nanoscale 2016, 8 (8), 4414-4435.

(3) Hu, F.; Zeng, C.; Long, R.; Miao, Y.; Wei, L.; Xu, Q.; Min, W. Supermultiplexed Optical Imaging and Barcoding with Engineered Polyynes. Nat. Methods 2018, 15 (3), 194-200.

(4) La Torre, A.; Botello-Mendez, A.; Baaziz, W.; Charlier, J. C.; Banhart, F. Strain-Induced Metal-Semiconductor Transition Observed in Atomic Carbon Chains. Nat. Commun. 2015, 6, 2-8.

(5) Cretu, O.; Botello-Mendez, A. R.; Janowska, I.; Pham-Huu, C.; Charlier, J. C.; Banhart, F. Electrical Transport Measured in Atomic Carbon Chains. Nano Lett. 2013, 13 (8), 3487-3493.

(6) Li, Y.; Sinitskii, A.; Tour, J. M. Electronic Two-Terminal Bistable Graphitic Memories. Nat. Mater. 2008, 7, 966.

(7) Standley, B.; Bao, W.; Zhang, H.; Bruck, J.; Lau, C. N.; Bockrath, M. Graphene-Based Atomic-Scale Switches. Nano Lett. 2008, 8 (10), 3345-3349.

(8) Hills, G.; Lau, C.; Wright, A.; Fuller, S.; Bishop, M. D.; Srimani, T.; Kanhaiya, P.; Ho, R.; Amer, A.; Stein, Y.; et al. Modern Microprocessor Built from Complementary Carbon Nanotube Transistors. Nature 2019, 572 (7771), 595-602.

(9) Ferrari, A. C.; Bonaccorso, F.; Fal'ko, V.; Novoselov, K. S.; Roche, S.; Bøggild, P.; Borini, S.; Koppens, F. H. L.; Palermo, V.; Pugno, N.; et al. Science and Technology Roadmap for Graphene, Related Two-Dimensional Crystals, and Hybrid Systems. Nanoscale 2015, 7 (11), 4598-4810.

(10) Artyukhov, V. I.; Liu, M.; Yakobson, B. I. Mechanically Induced Metal-Insulator Transition in Carbyne. Nano Lett. 2014, 14 (8), 4224-4229.

(11) Wang, M.; Lin, S. Ballistic Thermal Transport in Carbyne and Cumulene with Micron-Scale Spectral Acoustic Phonon Mean Free Path. Sci. Rep. 2016, 5, 18122.

(12) Lang, N. D.; Avouris, P. Oscillatory Conductance of CarbonAtom Wires. Phys. Rev. Lett. 1998, 81 (16), 3515-3518.

(13) Zanolli, Z.; Onida, G.; Charlier, J.-C. Quantum Spin Transport in Carbon Chains. ACS Nano 2010, 4 (9), 5174-5180.

(14) Baughman, R. H. Dangerously Seeking Linear Carbon. Science (Washington, DC, U. S.) 2006, 312 (5776), 1009-1110.

(15) Ravagnan, L.; Siviero, F.; Lenardi, C.; Piseri, P.; Barborini, E.; Milani, P.; Casari, C. S.; Li Bassi, A.; Bottani, C. E. Cluster-Beam Deposition and in Situ Characterization of Carbyne-Rich Carbon Films. Phys. Rev. Lett. 2002, 89 (28), 285506.

(16) Bonardi, P.; Achilli, S.; Tantardini, G. F.; Martinazzo, R Electron Transport in Carbon Wires in Contact with Ag Electrodes: A Detailed First Principles Investigation. Phys. Chem. Chem. Phys. 2015, 17 (28), 18413-18425.

(17) Xu, W.; Leary, E.; Hou, S.; Sangtarash, S.; González, M. T.; Rubio-Bollinger, G.; Wu, Q.; Sadeghi, H.; Tejerina, L.; Christensen, K. E.; et al. Unusual Length Dependence of the Conductance in Cumulene Molecular Wires. Angew. Chem., Int. Ed. 2019, 58 (25) $8378-8382$

(18) Milan, D. C.; Krempe, M.; Ismael, A. K.; Movsisyan, L. D.; Franz, M.; Grace, I.; Brooke, R. J.; Schwarzacher, W.; Higgins, S. J.; Anderson, H. L.; et al. The Single-Molecule Electrical Conductance of a Rotaxane-Hexayne Supramolecular Assembly. Nanoscale 2017, 9 (1), 355-361.

(19) Ballmann, S.; Hieringer, W.; Secker, D.; Zheng, Q.; Gladysz, J. A.; Görling, A.; Weber, H. B. Molecular Wires in Single-Molecule Junctions: Charge Transport and Vibrational Excitations. ChemPhysChem 2010, 11 (10), 2256-2260.

(20) Moreno-García, P.; Gulcur, M.; Manrique, D. Z.; Pope, T.; Hong, W.; Kaliginedi, V.; Huang, C.; Batsanov, A. S.; Bryce, M. R.; Lambert, C.; et al. Single-Molecule Conductance of Functionalized 
Oligoynes: Length Dependence and Junction Evolution. J. Am. Chem. Soc. 2013, 135 (33), 12228-12240.

(21) Ravagnan, L.; Piseri, P.; Bruzzi, M.; Miglio, S.; Bongiorno, G.; Baserga, A.; Casari, C. S.; Li Bassi, A.; Lenardi, C.; Yamaguchi, Y.; et al. Influence of Cumulenic Chains on the Vibrational and Electronic Properties of Sp-Sp2 Amorphous Carbon. Phys. Rev. Lett. 2007, 98 (21), 216103.

(22) Cumulenes. IUPAC Compend. Chem. Terminol. 2008, 1307, 2014. DOI: $10.1351 /$ goldbook.c01440.

(23) Januszewski, J. A.; Tykwinski, R. R. Synthesis and Properties of Long [n] Cumulenes $(n \geq 5)$. Chem. Soc. Rev. 2014, 43, 3184.

(24) Tykwinski, R. R.; Chalifoux, W.; Eisler, S.; Lucotti, A.; Tommasini, M.; Fazzi, D.; Del Zoppo, M.; Zerbi, G. Toward Carbyne: Synthesis and Stability of Really Long Polyynes. Pure Appl. Chem. 2010, 82, 891.

(25) Frankenberger, S.; Januszewski, J. A.; Tykwinski, R. R. Oligomers from Sp-Hybridized Carbon: Cumulenes and Polyynes. In Fullerenes and Other Carbon-Rich Nanostructures; Nierengarten, J.F., Ed.; Springer: Berlin, 2014; pp 219-256. DOI: 10.1007/ $430 \quad 2013$ 110.

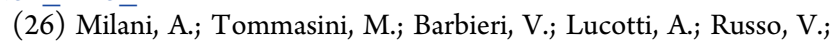
Cataldo, F.; Casari, C. S. Semiconductor-to-Metal Transition in Carbon-Atom Wires Driven by Sp2 Conjugated End Groups. J. Phys. Chem. C 2017, 121 (19), 10562-10570.

(27) Chynwat, V.; Coffin, T. L.; Wang, H.; Connors, R. E. Excited Electronic States of Arylbutatrienes. J. Phys. Chem. 1996, 100 (13), 5217-5223.

(28) Berkovitch-Yellin, Z.; Leiserowitz, L. Electron Density Distribution in Cumulenes: An X-Ray Study of Tetraphenylbutatriene at $20^{\circ} \mathrm{C}$ and $-160^{\circ} \mathrm{C}$. Acta Crystallogr., Sect. B: Struct. Crystallogr. Cryst. Chem. 1977, 33 (12), 3657-3669.

(29) Groom, C. R.; Bruno, I. J.; Lightfoot, M. P.; Ward, S. C. The Cambridge Structural Database. Acta Crystallogr., Sect. B: Struct. Sci., Cryst. Eng. Mater. 2016, 72 (2), 171-179.

(30) Milani, A.; Tommasini, M.; Russo, V.; Li Bassi, A.; Lucotti, A.; Cataldo, F.; Casari, C. S. Raman Spectroscopy as a Tool to Investigate the Structure and Electronic Properties of Carbon-Atom Wires. Beilstein J. Nanotechnol. 2015, 6, 480.

(31) Smith, J.; Hamilton, R.; Qi, Y.; Kahn, A.; Bradley, D. D. C.; Heeney, M.; McCulloch, I.; Anthopoulos, T. D. The Influence of Film Morphology in High-Mobility Small-Molecule:Polymer Blend Organic Transistors. Adv. Funct. Mater. 2010, 20 (14), 2330-2337.

(32) Sze, S. M.; Ng, K. K. Physics of Semiconductor Devices; Wiley, 2006. 